\section{Hochrisiko-Myelom: Prognose verbessern}

Die Einführung von Thalidomid, Lenalidomid und Bortezomib verbesserte die Prognose von Patienten mit multiplem Myelom (MM) dramatisch. Der relative Nutzen oder auch Schaden dieser "novel drugs" in den zytogenetisch heterogenen Subgruppen bleibt bisher unklar.

$\mathrm{O}$ ptimistische Schätzungen gehen heute von 5-Jahres-Überlebensraten für transplantierfähige Patienten von über $70 \%$ aus. Das Gesamtüberleben von Subgruppen mit „ungünstigem“ zytogenetischem Profil bleibt indes trotz Ausschöpfung aggressivster Therapieformen unbefriedigend. P. Leif Bergsagel und sein Team erachten es daher als notwendig, Behandlungsstrategien zu entwickeln, die auf die negativen prognostischen Faktoren abzielen und das Überleben dieser problematischen Patientengruppe verbessern.

Genetische Marker mit prognostischer Relevanz zu detektieren, erfordert entsprechendes High-Tech-Equipment und komplexe bioinformatische Analy- sen, die in den routinemäßigen klinischen Alltag noch nicht flächendeckend vorkommen. Als häufigste chromosomale Anomalien mit schlechterer Prognose dominieren IGH-Translokationen wie $\mathrm{t}(4 ; 14)$ und Deletion $17 \mathrm{p}$.

Der Vergleich des Outcome von Hochrisiko- vs. Standardrisiko-Patienten unter einer Therapie mit "novel drugs" stößt aufgrund meist geringer Fallzahlen an methodische Grenzen. In der Evaluierung des Erfolgs einer modernen, zielgerichteten Therapie unterscheiden die Autoren zwei Konzepte: Überwindung der ungünstigen Prognose - das Anheben des Gesamtüberlebens in der Hochrisiko-Gruppe auf das Niveau der Standard-Gruppe - und Verbesserung des Outcome - die Verbesserung der Überlebenszeit durch eine neue Therapiestrategie gegenüber einer Standardbehandlung in dieser Hochrisiko-Gruppe. In ihrem Review analysierten die Autoren Daten zur Behandlung des MM mit Thalidomid, Lenalidomid und Bortezomib. Ein wichtiges Ergebnis: Hochrisiko-Patienten scheinen von einer Bortezomib-Therapie zu profitieren, während die Erhaltungstherapie mit Thalidomid das Ergebnis verschlechtert.

Fazit: „Novel drugs“, die bei MM-Patienten ohne ungünstige zytogenetische Marker positiv wirken, könnten in Hochrisiko-Gruppen unwirksam sein oder sogar die Prognose verschlechtern. Daher sollten gepoolte Daten und weitere Studienergebnisse gesammelt und ausgewertet werden, so die Autoren.

Wolfgang Zimmermann

Bergsagel PL et al. Improving overall survival and overcoming adverse prognosis in the treatment of cytogenetically high-risk multiple myeloma. Blood. 2013;121(6):884-92.

\title{
Fragen der Lebensqualität im Vordergrund
}

\section{Die Prognose von Myelompatienten ist dank innovativer Therapieoptionen besser geworden. Daher rücken Aspekte der gesundheitsbezogenen Lebens- qualität in den Vordergrund. Welche Bedeutung haben Instrumente zur Messung der Lebensqualität in Studien und im klinischen Alltag.}

$\mathrm{M}$ yelompatienten scheinen stärker unter Krankheits- und Therapiebedingten Symptomen zu leiden als Patienten mit anderen hämatologischen Malignomen. Instrumente zur Erfassung der gesundheitsbezogen Lebensqualität (HRQoL) werden in klinischen Studien, gesundheitsökonomischen Evaluationen und im klinischen Alltag verwendet. Eingesetzt werden sie z. B. zur Prognostizierung, zum Monitoring des Therapieansprechens oder zur Verbesserung der Arzt-Patienten-Kommunikation.

Thomas R. Osborne und Kollegen screenten systematisch die aktuelle wissenschaftliche Literatur mit dem Ziel der Identifikation und Evaluierung möglichst aller publizierter HRQoL-Instrumente im Zusammenhang mit dem Krankheitsbild multiples Myelom. Da- bei wurden Fragestellungen aus Patientensicht ebenso berücksichtigt wie das Konzept des Messinstruments, dessen Eignung für die Erfassung aller patientenbezogenen Punkte und die Tauglichkeit für den Einsatz in unterschiedlichen Settings. Kein einziges Instrument adressierte alle patientenbezogenen Fragestellungen. Auch beschränkte sich die Validierung meist auf das Studienumfeld unter Aussparung des klinischen Alltags.

Fazit: Die nach Ansicht der Autoren besten in dieser systematischen Recherche gefunden Instrumente zur Beurteilung

Dringend gesucht: Instrumente zur Erfassung der gesundheitsbezogenen Lebensqualität für den klinischen Alltag. der HRQoL von Myelompatienten finden sich ausschließlich im Studienumfeld. Allerdings kommen patientenbezogene Fragestellungen zu kurz. Nützlich wäre die Entwicklung und Validierung von HRQoL-Instrumenten für den klinischen Einsatz. Wolfgang Zimmermann

Osborne TR et al. What issues matter most to people with multiple myeloma and how well are we measuring them? A systematic review of quality of life tools. Eur J Haematol. 2012;89(6): 437-57.

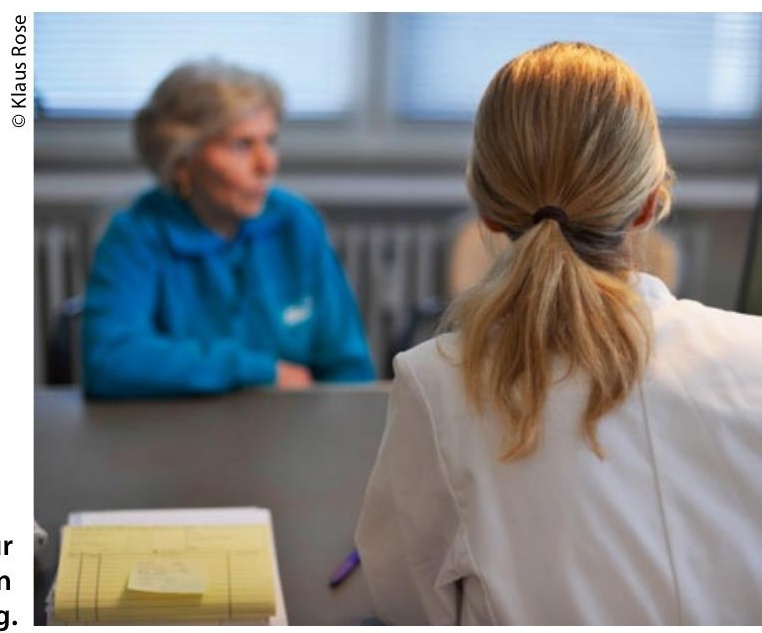

